\title{
Provoking thoughts on agriculture
}

\section{J.C. Bowman}

Agricultural Research Policy. By Vernon W. Ruttan.

University of Minnesota Press: 1982. Pp.369. Hbk \$32.50; pbk \$13.95.

IN an admirable introduction Professor Ruttan presents his credentials for examining agricultural research policy. For 30 years he has been employed as an economist, mostly in the United States but also in the Philippines and Japan. In recent years the emphasis of his interests has, he says, moved from economics to agricultural research policy, about which he has been a prolific writer.

Professor Ruttan's introduction concludes:

In the book judgements are made about agricultural research policy. These judgements are not confined by any formal philosophical system. They arise out of a combination of scientific analysis, professional experience and my personal perception of responsibility for the results of scientific inquiry and technological development.

He has remained firm to his brief and has not formally reviewed the literature on the subject; indeed there are only a few indications in the text that others may not agree with Professor Ruttan on many of the matters which he discusses. At this stage the reader might be forgiven for some lack of trust in the author's integrity.

Each of the chapters is usually based on one of Professor Ruttan's previous publications or lectures. Whilst the writing is lucid the integration of source material might have been more thorough, reducing the impression that each chapter is an unrelated aspect of a common theme. Another, more serious, criticism is that the distinction between Professor Ruttan's idea of agricultural research policy in general is not always clearly dissociated from his views about American practices. The suspicion lingers until near the end of the book that the author harbours some hope of finding a single optimum solution to the determination and management of agricultural research policy, and that this is not far removed from the current approach in the United States. The last two chapters, however, happily prove such suspicion to be unfounded and make clear the author's continuing pursuit of alternative answers in an international context.

The main purposes of agricultural research might be said to be four-fold: to improve the return on resources used in existing methods of production, processing, distribution, marketing and consumption; to make the final product more acceptable to the consumer in quality and price; to make the existing methods of production etc. more acceptable on social and moral grounds; and to facilitate change in existing processes to meet future needs and opportunities. Such a statement does not appear in Professor Ruttan's book. Rather he takes these objectives for granted and discusses the achievements of agricultural research in increasing agricultural output per unit of individual and total resource inputs. This is a familiar economic approach. He then tackles some of the factors determining the efficiency of agricultural research, such as the nature of the research institution, national and international systems (with a rather brief reference to Europe and the role of FAO), its funding, and its location and scale.

Professor Ruttan's approach to these topics is somewhat mechanistic. Whilst it is clear that he appreciates the importance of the context in which research is conducted, the effect of the motives of individual scientists on the pertinence and success of a research programme is largely ignored. The desire of research workers to pursue knowledge for its own sake may not always coincide with the objectives held by those who invest in research, and here it would have been interesting to have the author's thoughts on the best means of selecting research managers and scientists, and how best to motivate them.

In considering the respective merits of public and private funding and responsibility, Professor Ruttan sets down the criteria, as he sees them, for the appropriate roles of each in agricultural research. The public sector has been justified, he says, because "in many areas incentives for private sector research have not been adequate to induce an optimum level of research investment", because of "its complementarity with education" and because "it has contributed to the maintenance or enhancement of a competitive structure in the agricultural production, input, and marketing sectors"'. Where, however, are the arguments that public sector research is needed to safeguard society's interests and to undertake basic research for which the private sector will rarely pay, however much money it has to invest? Did Professor Ruttan omit them because he did not consider them important and because he did not consider them valid within his perceived scheme for an improved agricultural research system?

Another interesting topic discussed at length by Professor Ruttan is the selection of a research portfolio. The peer review system, cost-benefit analysis as well as more sophisticated systems for choosing a research programme are considered. But where are the deficiencies and long-term biases of the peer review method, how can the allocation of funds be determined for basic research, when by definition the results of the research are not known, and how should the appropriateness of research be judged?

For the reader studying agricultural research policy for the first time this book would not be a good starting point because of its selective, at times self-indulgent, air. Conversely, those familiar with the topic will find the book both provocative and stimulating. In the end even the author is left asking questions about the role of social science in agricultural research, about the responsibility to society of research scientists and about the part nonscientists may play in determining the amount and type of research undertaken. It is a measure of the thought-provoking nature of Professor Ruttan's book that one would like to discuss these issues with him.

J.C. Bowman, formerly Director of the Centre for Agricultural Strategy at Reading University, is Secretary to the Natural Environment Research Council.

\section{From fantasy to fact}

\section{Fred Rosen}

\section{Monoclonal Antibodies in Clinical} Medicine.

Edited by Andrew J. McMichael and John W. Fabre.

Academic: 1982. Pp.661. £34, \$63

IN THEIR paper submitted to Nature on 14 May 1975 Köhler and Milstein concluded: "Such cells can be grown in vitro in massive cultures to provide specific antibody. Such cultures could be valuable for medical and industrial use" (Nature 256, 495; 1975). That may prove to be the scientific understatement of the century. Hybridoma technology has fructified to such an extent as to have become one of the keystones of the biological revolution in progress.

McMichael and Fabre have assembled a notable group of contributors to a volume that lucidly shows what hybridomas have done for medicine and biology. This book is a salubrious mixture of fact and fantasy from its introduction by Milstein, who describes the simple and beautiful experiments that produced this wonder, to the rich speculations of H.S. Kaplan et al. on what life might be like if only we had a thesaurus of human hybridomas.

As one proceeds through the plethora of contributions to immunology, haematology, genetics, virology, parasitology, bacteriology, neurobiology and oncology, each of which is supported by graphs, tables and photomicrographs, the reader comes to ponder: "Why monoclonal antibodies? Why not goat or rabbit antibodies?". Near the end of this volume the answer is brilliantly presented in a chapter by E. Haber. With the seemingly trivial aim of discussing monoclonal antibodies to drugs such as digoxin or biologically active 